\title{
Comparison of the Effects of Lidocaine and Dexmedetomidine Before Propofol Induction During Laryngeal Mask Airway Insertion
}

\author{
(D) Ali Sait Kavaklı1, (D) Dilek Kitapçığlu² \\ 1 University of Health Sciences, Antalya Training and Research Hospital, Clinic of Anaesthesiology and Reanimation, Antalya, Turkey \\ ${ }^{2}$ Acıbadem Mehmet Ali Aydınlar University Faculty of Medicine, Department of Medical Education, Istanbul, Turkey
}

\section{Abstract}

Objective: Laryngeal mask (LMA) is an airway device that can be used as an alternative to face mask and tracheal intubation. Different drug combinations can be used to ensure suitable conditions during LMA insertion. Lidocaine and dexmedetomidine are drugs that can be used to reduce hemodynamic response and suppress oropharyngeal reflexes during both intubation and LMA insertion. The aim of this study was to investigate the effects of lidocaine and dexmedetomidine on LMA insertion.

Methods: Sixty patients who were scheduled to undergo cystoscopy under general anesthesia with LMA were included in the study. Patients were randomly divided into two groups: Group L (those who received $1.5 \mathrm{mg} / \mathrm{kg}$ lidocaine) and Group D (those who received $1 \mu \mathrm{g} / \mathrm{kg}$ dexmedetomidine). Number of attempts of LMA insertion, ease of LMA insertion, mouth opening, laryngospasm, gumming, stomach distention, limb movements and spontaneous breathing and hemodynamic parameters were recorded.

Results: There was no statistically significant difference between the two groups in terms of the number of attempts of LMA insertion. In the first attempt, the LMA insertion success rate was 70\% for Group L and 73.3\% for Group D. There was no statistically significant difference between the two groups in terms of mouth opening, laryngospasm, ease of LMA insertion, gumming, stomach distension, limb movement and spontaneous breathing. Systolic blood pressure, diastolic blood pressure, mean blood pressure and heart rate were statistically lower in Group D after drug administration. It was determined that the decreases in the other times were statistically significant compared to the baseline values after drug administration in both groups.

Conclusion: Based on the results of the present study, $1 \mu \mathrm{g} / \mathrm{kg}$ dexmedetomidine and $1.5 \mathrm{mg} / \mathrm{kg}$ lidocaine used before propofol induction provided similar conditions for LMA insertion. Dexmedetomidine has a more hypotensive effect and provides a greater reduction in heart rate than lidocaine. Furthermore, the use of lidocaine before propofol induction provides better hemodynamic control than dexmedetomidine.

Keywords: Laryngeal mask airway, lidocaine, dexmedetomidine

\section{INTRODUCTION}

Laryngeal mask (LMA) is an airway device that can be used as an alternative to facial mask and tracheal intubation. Although there are studies showing that LMA requires less anesthesia compared to endotracheal intubation (1), adequate depth of anesthesia should be provided for proper LMA insertion. It has been reported that the use of propofol in induction provides better conditions for LMA insertion than thiopental because of its higher depressant effects on jaw relaxation and airway reflexes $(2,3)$. However, the use of propofol alone without premedication may be insufficient for LMA insertion. Increasing the dose of propofol to ensure proper conditions increases the incidence of undesirable effects such as cardiac depressant effects $(4,5)$. Lidocaine is an agent that can be used both topically and intravenously because of its dose-dependent suppressing effects on cardiovascular responses and cough reflex due to intubation and LMA insertion (6,7). In addition, lidocaine use has been reported to cause a decrease in the incidence of laryngospasm (8). There are studies showing that dexmedetomidine, a selective $\alpha 2$-receptor agonist, reduces respiratory and cardiovascular responses during intubation and extubation $(9,10)$. In addition, dexmedetomidine has been reported to be a suitable agent for LMA insertion (11). The aim of this study was to compare the 
effects of lidocaine and dexmedetomidine on LMA insertion administered before propofol induction.

\section{METHODS}

This study was prepared in accordance with the Declaration of Helsinki. It was approved by the ethics committee and carried out at the Clinic of Anesthesiology and Reanimation, Taksim Training and Research Hospital, Ministry of Health. Written informed consent was obtained from all patients. The primary aim of this study was to investigate the effects of lidocaine and dexmedetomidine administered before induction on LMA insertion quality in patients undergoing cystoscopy. The secondary objective was to investigate the effects of lidocaine and dexmedetomidine on hemodynamic parameters. Sixty American Society of Anaesthesiologists (ASA) I-II patients aged between 40-75 years who were scheduled for cystoscopy under general anesthesia with LMA were included in the study. Patients were randomly divided into two groups: Group L (lidocaine-treated group) and Group D (dexmedetomidinetreated group). Patients with ASA III and above, a history of bleeding diathesis, gastroesophageal reflux disease, a history of previous head and neck surgery, and cooperative impairment were excluded. Standard anesthesia monitoring including pulse oximetry, electrocardiography and noninvasive blood pressure measurement was performed to patients who were taken to the operating room following preoperative routine anesthesia preparation. Peripheral venous access was established with 20 $\mathrm{G}$ cannula and $0.09 \% \mathrm{NaCl}$ infusion was started at $2.0 \mathrm{~mL} / \mathrm{kg} / \mathrm{h}$. Following preoxygenation, $1.5 \mathrm{mg} / \mathrm{kg}$ intravenous (IV) lidocaine diluted with $20 \mathrm{~mL}$ saline was administered to Group L and 1 $\mathrm{mcg} / \mathrm{kg}$ IV dexmedetomidine diluted with $20 \mathrm{~mL}$ saline was administered to Group D within 2 minutes. Anesthesia was then induced with $2.5 \mathrm{mg} / \mathrm{kg}$ propofol and $1 \mathrm{mcg} / \mathrm{kg}$ fentanyl. Neuromuscular blocking agent was not used for muscle relaxation. The first trial was performed for LMA insertion 90 seconds after anesthesia induction. Lubricant gel was used for LMA insertion. Following LMA insertion, the cuff was inflated with the recommended volumes and ventilation was confirmed by end-tidal carbon dioxide measurement. If the first attempt failed, the second trial was performed after 30 seconds of mask ventilation. Patients with three failed attempts were excluded. Anesthesia was maintained with $1.5 \%$ sevoflurane in mechanically ventilated patients with $45 \% \mathrm{O}_{2}-65 \% \mathrm{~N}_{2} \mathrm{O}$ mixture. All LMA insertion procedures were performed by the same person. Evaluations were performed by an anesthesiologist independent of the study. Ease of LMA insertion (easy, difficult, impossible), mouth opening (complete, partial, no), gag reflex (yes, no), stomach distention (yes, no), limb movement (yes, no), spontaneous breathing (yes, no) and laryngospasm (yes, no) were recorded. Laryngospasm was defined as the presence of stridor lasting more than 15 seconds and absence of a capnography wave in the absence of any other upper airway obstruction during LMA insertion. Systolic artery pressure (SAP), diastolic artery pressure (DAP), mean arterial pressure (MAP) and heart rate (HR) values were recorded at baseline, 90 seconds before LMA insertion and 1, 3, 5, 10 and 15 minutes after LMA insertion.

\section{Statistical Analysis}

SPSS version 15 statistical software (SPSS Inc., Chicago, IL, USA) was used for statistical evaluation of the data. Descriptive statistics (mean, standard deviation, frequency, percentage) were used, and qualitative data were compared using Pearson chisquare and Fisher's exact tests. Mann-Whitney $U$ test was used to compare the quantitative data. The Wilcoxon sign test was used for intragroup comparisons of quantitative data. Results were evaluated at $95 \%$ confidence interval and $p<0.05$ level of significance.

\section{RESULTS}

Sixty patients who underwent elective cystoscopy under general anesthesia with LMA were included in the study. Surgical procedures were completed with LMA under general anesthesia in all patients. None of the patients had respiratory complications requiring intubation. There was no statistically significant difference between the two groups in terms of age, weight, gender, ASA score and mallampati scores. Demographic data of the patients are presented in Table 1. No statistically

\begin{tabular}{|c|c|c|c|}
\hline & Group L $(n=30)$ & Group D $(n=30)$ & $\mathbf{p}$ \\
\hline Age (years) & $56.37 \pm 8.93$ & $55.67 \pm 9.59$ & 0.824 \\
\hline Weight (kg) & $74 \pm 12.2$ & $69.87 \pm 9.64$ & 0.088 \\
\hline \multicolumn{4}{|l|}{ Gender } \\
\hline Female & $8(26.7)$ & $4(13.3)$ & \multirow{2}{*}{0.197} \\
\hline Male & $22(73.3)$ & $26(86.7)$ & \\
\hline \multicolumn{4}{|l|}{ ASA score } \\
\hline ASA I & $13(43.3)$ & $20(66.7)$ & \multirow{2}{*}{0.06} \\
\hline ASA II & $17(56.7)$ & $10(33.3)$ & \\
\hline \multicolumn{4}{|c|}{ Mallampati score } \\
\hline I & $15(50)$ & $22(73.3)$ & \multirow{3}{*}{0.077} \\
\hline II & $12(40)$ & $8(26.7)$ & \\
\hline III & $3(10)$ & $0(0)$ & \\
\hline
\end{tabular}


significant difference was found between the two groups in terms of LMA insertion trials. LMA insertion success was $70 \%$ for Group L and 73.3\% for Group D in the first attempt. In Group D, LMA insertion was performed in the third attempt in one patient (3.3\%), and in all patients, the first and second trials were successful. For Group L, LMA insertion was performed in all patients in the first and second trials. When mouth opening, laryngospasm, ease of LMA insertion, gagging, gastric distension, limb movement and spontaneous breathing findings were evaluated, there was no statistically significant difference between the two groups (Table 2). While there was no statistically significant difference between the basal values of SAP, DAP and MAP in both groups, values were significantly lower in Group D compared to Group L after drug administration. When SAP, DAP and MAP changes of the groups were compared, it was seen that there were significant decreases in both groups compared to baseline values. Intergroup comparisons revealed a

\begin{tabular}{|c|c|c|c|}
\hline & Group L $(n=30)$ & Group D $(n=30)$ & $p$ \\
\hline \multicolumn{4}{|c|}{ Number of LMA insertion trials } \\
\hline 1 & $21(70)$ & $22(73.3)$ & \multirow{3}{*}{0.529} \\
\hline 2 & $9(30)$ & $7(23.3)$ & \\
\hline 3 & $0(0)$ & $1(3.3)$ & \\
\hline \multicolumn{4}{|c|}{ LMA insertion ease } \\
\hline Easy & $19(63.3)$ & $24(80)$ & \multirow{2}{*}{0.152} \\
\hline Difficult & $11(36.7)$ & $6(20)$ & \\
\hline \multicolumn{4}{|c|}{ Mouth opening } \\
\hline Complete & $17(56.7)$ & $21(70)$ & \multirow{2}{*}{0.284} \\
\hline Partial & $13(43.3)$ & $9(30)$ & \\
\hline \multicolumn{4}{|c|}{ Laryngospasm } \\
\hline No & $29(96.7)$ & $29(96.7)$ & \multirow{2}{*}{0.754} \\
\hline Yes & $1(3.3)$ & $1(3.3)$ & \\
\hline \multicolumn{4}{|l|}{ Gagging } \\
\hline No & $26(86.7)$ & $29(96.7)$ & \multirow{2}{*}{0.177} \\
\hline Yes & $4(13.3)$ & $1(3.3)$ & \\
\hline \multicolumn{4}{|c|}{ Stomach distension } \\
\hline No & $26(86.7)$ & $27(90)$ & \multirow{2}{*}{0.500} \\
\hline Yes & $4(13.3)$ & $3(10)$ & \\
\hline \multicolumn{4}{|c|}{ Limb movement } \\
\hline No & $18(60)$ & $16(53.3)$ & \multirow{2}{*}{0.602} \\
\hline Yes & $12(40)$ & $14(46.7)$ & \\
\hline \multicolumn{4}{|c|}{ Spontaneous breathing } \\
\hline No & $15(50)$ & $11(36.7)$ & \multirow{2}{*}{0.297} \\
\hline Yes & $15(50)$ & $19(63.3)$ & \\
\hline
\end{tabular}

statistically significant decrease in all values measured after drug administration compared to baseline in Group L. Similarly, there was a statistically significant decrease in Group D compared to baseline values after drug administration. Significant increases in SAP, DAP and MAP were observed in both groups 3 minutes after LMA insertion. HRs were significantly lower in Group $L$ at all times than in Group D (Figure 1).

\section{DISCUSSION}

Our study showed that the administration of lidocaine and dexmedetomidine before induction had similar effects on the quality of LMA insertion in patients undergoing cystoscopy, and that dexmedetomidine had more effect on hemodynamic parameters than lidocaine. Dexmedetomidine is a selective $\alpha 2$ agonist with analgesic and sedative effects. There are studies that dexmedetomidine reduces the respiratory and circulatory stimuli in intubation and extubation. Wei et al. (12) reported that dexmedetomidine at a dose of $1 \mu \mathrm{g} / \mathrm{kg}$ improves intubation conditions in children and suppresses the hemodynamic response due to intubation. It has also been reported that dexmedetomidine at a dose of $0.6 \mu \mathrm{g} / \mathrm{kg}$ significantly reduces hemodynamic responses to laryngoscopy and endotracheal intubation in patients undergoing thyroid surgery (13). It has been shown that dexmedetomidine at a dose of $1 \mu \mathrm{g} / \mathrm{kg}$ given 30 seconds before propofol induction provides optimum conditions for LMA insertion at 90 seconds after induction and that propofol-dexmedetomidine combination is more effective on LMA insertion than the propofol-fentanyl combination (11). In addition, there are also studies showing that dexmedetomidine facilitates LMA insertion and reduces propofol requirement (14). Similarly, there are studies examining the effects of lidocaine on intubation and LMA insertion. Kocamanoglu et al. (15) reported that both IV and topical lidocaine limited the hemodynamic response in laryngoscopy and endotracheal intubation. Hashemian et al. (16) reported that the combination of fentanyl and lidocaine is more effective than the use of fentanyl alone to prevent hemodynamic response due to intubation. In their study examining the effects of lidocaine on LMA insertion, Baik et al. (17) detected that $1.5 \mathrm{mg} / \mathrm{kg}$ dose of lidocaine did not make hemodynamic changes, although the incidence of cough, gagging and laryngospasm was found to be decreased compared to the control group. In our study, similar rates were observed in the incidence of gagging and laryngospasm in both groups. These low rates indicate the utility of both agents in LMA insertion. At the same time, less hemodynamic response in the lidocaine group than dexmedetomidine group was consistent with Baik et al. (17) The authors also reported the success rate 
8AB

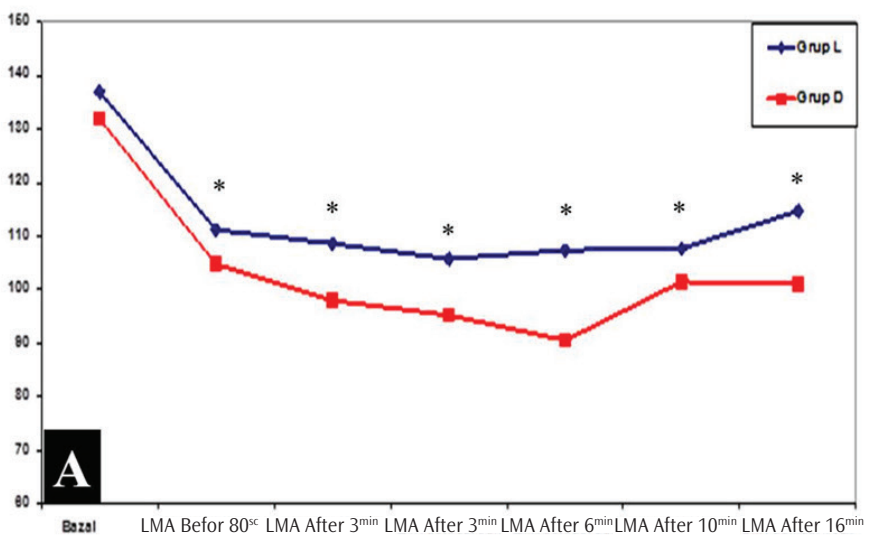

$O A B$

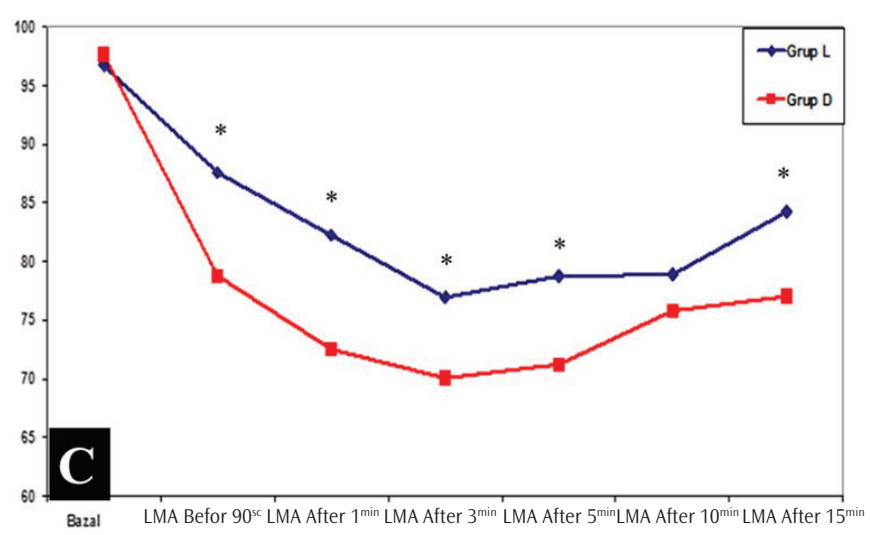

DAB

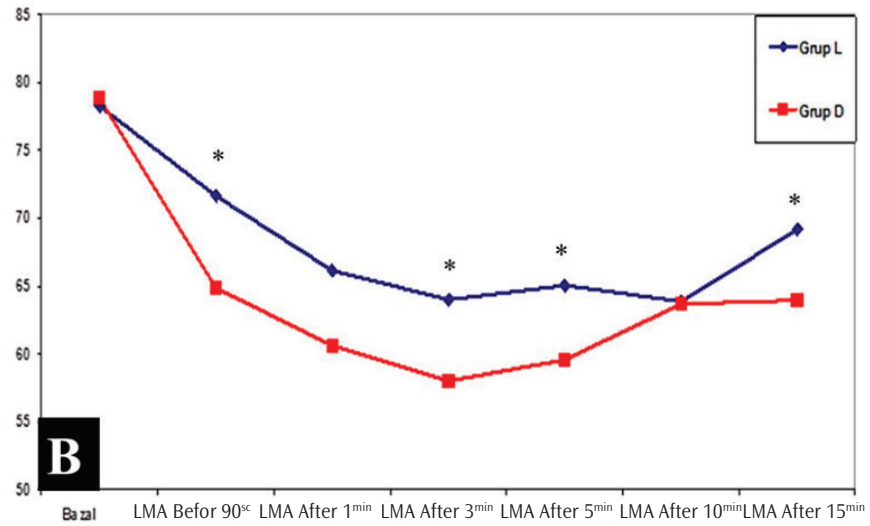

KAH

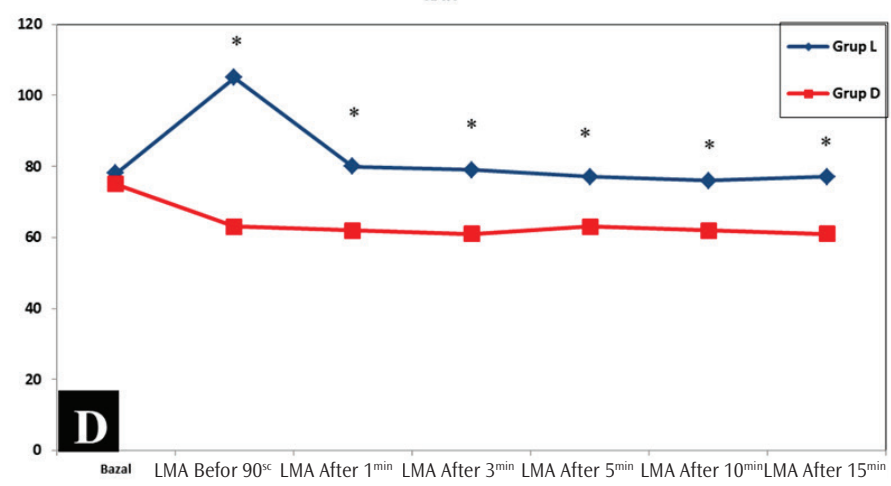

Figure 1. A) Variation of systolic arterial pressure of groups with time, B) Variation of diastolic arterial pressure of groups with time, C) Variation of mean arterial pressure of groups with time, D) Variation of heart rate of groups with time

${ }^{*} p<0.05$ comparison between groups

LMA: Laryngeal mask

of LMA insertion to be $97.5 \%$ in the lidocaine group versus the $85 \%$ success rate in the control group. In our study, the $100 \%$ success rate seen in the lidocaine group after a maximum of two trials confirms the results of this study. Although there is no study comparing the effects of both agents on intubation or LMA insertion quality in the literature, there are studies showing that the use of two agents together provides better conditions. Hancl et al. (18) reported that dexmedetomidine-lidocaine-propofol combination provides better intubation conditions than fentanyl-lidocaine-propofol combination. In the study of Yoo et al. (19), $1 \mathrm{mg} / \mathrm{kg}$ dose of dexmedetomidine together with $0.5 \mathrm{mg} /$ $\mathrm{kg}$ lidocaine reduced propofol requirement by $38 \%$. Similarly, both agents reduced the need for sevoflurane; however, it was reported that patients receiving dexmedetomidine had less need of sevoflurane than those receiving lidocaine (20).

Consistent with all these studies, both lidocaine and dexmedetomidine were found to be effective in LMA insertion in our study. After a maximum of two attempts, $100 \%$ success rate was achieved with lidocaine use and this rate was $96.6 \%$ for dexmedetomidine. The low incidence of gagging, gastric distension and laryngospasm with both agents indicates that these agents can be used safely in LMA insertion. Although there is no definite conclusion about the appropriate time for LMA insertion after propofol induction, some studies have reported some time periods for jaw relaxation and proper mouth opening. Goyagi et al. (21) studied the effect of the use of fentanyl on LMA insertion and they used lidocaine to prevent injection pain 30 seconds before propofol induction and reported that appropriate conditions were provided for LMA insertion in 90 seconds after propofol induction. Uzumcugil et al. (11) performed induction with propofol 30 seconds after injection of $1 \mu \mathrm{g} / \mathrm{kg}$ dexmedetomidine and reported successful insertion of LMA 90 seconds after induction. Similarly, Baik et al. (17) reported appropriate conditions for LMA insertion were achieved within 90 seconds after lidocaine injection during 
anesthesia induction. In all of these studies, it was concluded that 90 seconds is sufficient to provide ideal jaw relaxation and mouth opening for LMA insertion after both dexmedetomidine and lidocaine injection. In our study, the first LMA insertion trial was performed in 90 seconds after propofol in accordance with these studies. Consistent with the previous studies, we found that the jaw relaxation and mouth opening were appropriate for LMA insertion in 90 seconds after both propofol lidocaine and propofol dexmedetomidine combinations. The sympatholytic effect of dexmedetomidine is dominant at low plasma concentrations. It leads to vasodilatation by activation of $\alpha 2$ receptors in the central nervous system and vascular endothelial cells, resulting in a decrease in MAP and HR. At high concentrations, a peripheral vasoconstrictive effect occurs with the effect of $\alpha 2$ adrenoreceptor activation in vascular smooth muscle cells, which may lead to an increase in MAP and a further decrease in HR $(22,23)$. Talke et al. (22) showed that the vasoconstrictive effect of dexmedetomidine continued while the sympatholytic effect decreased under general anesthesia. However, suppressing or reversing the increase in hemodynamic effects by the presence of concomitant comorbidity or the use of dexmedetomidine in combination with drugs remains unclear. In studies conducted on healthy volunteers, it has been reported that there is a $21 \%$ and $31 \%$ decrease in HR after two minutes dexmedetomidine infusion at a dose of $1-2 \mu \mathrm{g} / \mathrm{kg}$ (24). The same study reports an increase in MAP of $7 \%$ and $8 \%$ at these doses. In contrast to this finding, in our study, the hypotensive response seen at doses of $1 \mu \mathrm{g} / \mathrm{kg}$ seems to be consistent with the hypothesis of suppressing or reversing the hemodynamic effects associated with concomitant use of dexmedetomidine and propofol. However, although there are studies showing the hypotensive effect of lidocaine (25), there are also studies showing that it is not associated with significant hemodynamic changes after endotracheal intubation (26). In our study, the fact that dexmedetomidine had more hypotensive effect and lower HRs than lidocaine treated group supported the opinion that lidocaine may provide a more stable hemodynamics after dexmedetomidine after LMA insertion. The limitation of our study is the absence of a control group in which propofol is used alone. However, we thought that the use of propofol for LMA insertion alone would have to increase the dose (19-27), which may lead to undesired respiratory and hemodynamic responses.

\section{CONCLUSION}

The use of $1 \mu \mathrm{g} / \mathrm{kg}$ dexmedetomidine and $1.5 \mathrm{mg} / \mathrm{kg}$ lidocaine before propofol induction creates similar and favorable conditions for LMA insertion. However, dexmedetomidine used before propofol induction has more hypotensive effect than lidocaine and causes more decrease in HR. The use of lidocaine before propofol induction provides better hemodynamic control than dexmedetomidine. Controlled randomized trials with larger patient groups may support the results of our study.

\section{Ethics}

Ethics Committee Approval: The study was approved by the Institutional Review Board, the study was conducted in accordance with the principles of the Helsinki Declaration.

Informed Consent: Written informed consent was obtained from all participants

Peer-review: External and internal peer-reviewed.

\section{Authorship Contributions}

Surgical and Medical Practices: A.S.K., D.K., Concept: A.S.K., D.K., Design: A.S.K., D.K., Data Collection or Processing: A.S.K., Analysis or Interpretation: A.S.K., D.K., Literature Search: A.S.K., D.K., Writing: A.S.K., D.K.

Conflict of Interest: The authors have no conflicts of interest to declared.

Financial Disclosure: The authors declared that this study has received no financial support.

\section{REFERENCES}

1. Wilkins CJ, Cramp PG, Staples J, Stevens WC. Comparison of the anesthetic requirement for tolerance of laryngeal mask airway and endotracheal tube. Anesth Analg 1992;75:794-7.

2. Brown GW, Patel N, Ellis FR. Comparison of propofol and thiopentone for laryngeal mask insertion. Anaesthesia 1991;46:771-2.

3. Scanlon P, Carey M, Power M, Kirby F. Patient response to laryngeal mask insertion after induction of anaesthesia with propofol or thiopentone. Can J Anaesth 1993;40:816-8.

4. Goh PK, Chiu CL, Wang CY, Chan YK, Loo PL. Randomized double-blind comparison of ketamine-propofol, fentanyl-propofol and propofol-saline on haemodynamics and laryngeal mask airway insertion conditions. Anaesth Intensive Care 2005;33:223-8.

5. Siddik-Sayyid SM, Aouad MT, Taha SK, Daaboul DG, Deeb PG, Massouh FM, et al. A comparison of sevoflurane-propofol versus sevoflurane or propofol for laryngeal mask airway insertion in adults. Anesth Analg 2005;100:1204-9.

6. Lee MC, Absalom AR, Menon DK, Smith HL. Awake insertion of the laryngeal mask airway using topical lidocaine and intravenous remifentanil. Anaesthesia 2006:61:32-5.

7. Erb TO, von Ungern-Sternberg BS, Keller K, Frei FJ. The effect of intravenous lidocaine on laryngeal and respiratory reflex responses in anaesthetised children*. Anaesthesia 2013;68:13-20.

8. Koc C, Kocaman F, Aygenc E, Ozdem C, Cekic A. The use of preoperative lidocaine to prevent stridor and laryngospasm after tonsillectomy and adenoidectomy. Otolaryngol Head Neck Surg 1998;118:880-2. 
9. Guler G, Akin A, Tosun Z, Eskitascoglu E, Mizrak A, Boyaci A. Single-dose dexmedetomidine attenuates airway and circulatory reflexes during extubation. Acta Anaesthesiol Scand 2005;49:1088-91.

10. Maroof M, Khan RM, Jain D, Ashraf M. Dexmedetomidine is a useful adjunct for awake intubation. Can J Anaesth 2005;52:776-7.

11. Uzumcugil F, Canbay O, Celebi N, Karagoz AH, Ozgen S. Comparison of dexmedetomidine-propofol vs. fentanyl-propofol for laryngeal mask insertion. Eur J Anaesthesiol 2008;25:675-80.

12. Wei L, Deng X, Sui J, Wang L, Liu J. Dexmedetomidine Improves Intubating Conditions Without Muscle Relaxants in Children After Induction With Propofol and Remifentanil. Anesth Analg 2015;121:785-90.

13. Rashmi HD, Komala HK. Clinical evaluation of the effect of intravenous dexmedetomidine on the hemodynamic response to laryngoscopy and endotracheal intubation in patients undergoing thyroid surgeries. Anesth Essays Res 2016;10:483-7.

14. Nellore SS, Waychal AD, Rustagi PS. Comparison of DexmedetomidinePropofol versus Fentanyl-Propofol on Insertion Conditions of Proseal Laryngeal Mask Airway. J Clin Diagn Res 2016;10:UC06-UC9.

15. Kocamanoglu IS, Cengel Kurnaz S, Tur A. Effects of lignocaine on pressor response to laryngoscopy and endotracheal intubation during general anaesthesia in rigid suspension laryngoscopy. J Laryngol Otol 2015;129:7985.

16. Hashemian AM, Zamani Moghadam Doloo H, Saadatfar M, Moallem R, Moradifar M, Faramarzi R, et al. Effects of intravenous administration of fentanyl and lidocaine on hemodynamic responses following endotracheal intubation. Am J Emerg Med 2018;36:197-201.

17. Baik HJ, Kim YJ, Kim JH. Lidocaine given intravenously improves conditions for laryngeal mask airway insertion during propofol target-controlled infusion. Eur J Anaesthesiol 2009;26:377-81.

18. Hanci V, Erdoğan G, Okyay RD, Yurtlu BS, Ayoğlu H, Baydilek Y, Turan IO. Effects of fentanyl-lidocaine-propofol and dexmedetomidine-lidocaine- propofol on tracheal intubation without use of muscle relaxants. Kaohsiung J Med Sci 2010;26:244-50.

19. Yoo JY, Kwak HJ, Kim YB, ParkCK, Lee SY, Kim JY. The effect of dexmedetomidine pretreatment on the median effective bolus dose of propofol for facilitating laryngeal mask airway insertion. J Anesth 2017;31:11-7.

20. Harsoor SS, Rani D, Roopa MN, Lathashree S, Sudheesh K, Nethra SS. "Anesthetic Sparing Effect of Intraoperative Lignocaine or Dexmedetomidine Infusion on Sevoflurane during General Anesthesia". Middle East J Anaesthesiol 2015;23:301-7.

21. Goyagi T, Tanaka M, Nishikawa T. Fentanyl decreases propofol requirement for laryngeal mask airway insertion. Acta Anaesthesiol Scand 2003;47:771-4.

22. Talke P, Lobo E, Brown R. Systemically administered alpha2-agonist-induced peripheral vasoconstriction in humans. Anesthesiology 2003;99:65-70.

23. Snapir A, Posti J, Kentala E, Koskenvuo J, Sundell J, Tuunanen H, et al. Effects of low and high plasma concentrations of dexmedetomidine on myocardial perfusion and cardiac function in healthy male subjects. Anesthesiology 2006;105:902-10.

24. Colin PJ, Hannivoort LN, Eleveld DJ, Reyntjens K, Absalom AR, Vereecke HEM, et al. Dexmedetomidine pharmacodynamics in healthy volunteers: 2 . Haemodynamic profile. Br J Anaesth 2017;119:211-20.

25. Totonchi Z, Salajegheh S, Mohaghegh MR, Kiaei MM, Shirvani M, Ghorbanlo M. Hemodynamic effect of intravenous lidocaine during aortic cannulation in cardiac surgery. Interv Med Appl Sci 2017;9:56-60.

26. Lin CC, Yu JH, Lin CC, Li WC, Weng YM, Chen SY. Postintubation hemodynamic effects of intravenous lidocaine in severe traumatic brain injury. Am J Emerg Med 2012;30:1782-7.

27. Kodaka M, Okamoto Y, Koyama K, Miyao H. Predicted values of propofol EC50 and sevoflurane concentration for insertion of laryngeal mask Classic and ProSeal. Br J Anaesth 2004;92:242-5. 